Supplementary Information

\title{
Computational Examination on the Active Site Structure of (peroxo)diiron(III) intermediate in the Amine oxygenase, AurF
}

Prabha Jayapal*, Azaj Ansari and Gopalan Rajaraman*

Department of chemistry, Indian Institute of Technology-Bombay, Powai, Mumbai. 
Figure S1: Optimized geometries of the quantum chemical cluster models investigated through B3LYP functional. Only hydrogen involved in hydrogen bonding are shown.

\section{Peroxo models:}

M1. $\mu-\eta^{2}: \eta^{2}$-peroxo- $\mathrm{Fe}_{2}{ }_{2}^{\text {IIIII }}$

(Numbering as per reference 1).

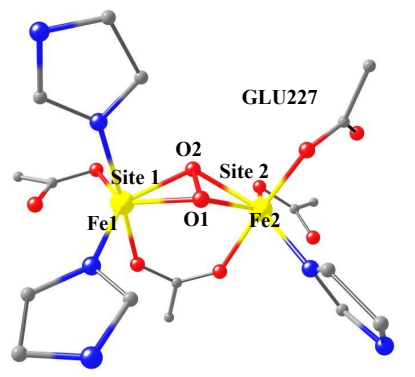

Peroxo models with Protonated glutamate:

227 M1. $\mu-\eta^{2}: \eta^{2}$-peroxo- $\mathrm{Fe}_{2}{ }^{\text {IIIIII }}$ with glutamate 227 protonated

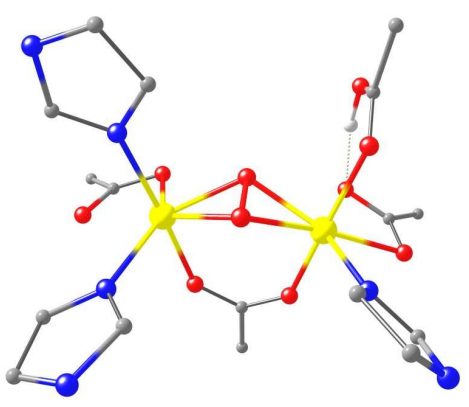

M2. $\mu$-1,1- peroxo- $\mathrm{Fe}_{2}{ }^{\text {III/III }}$

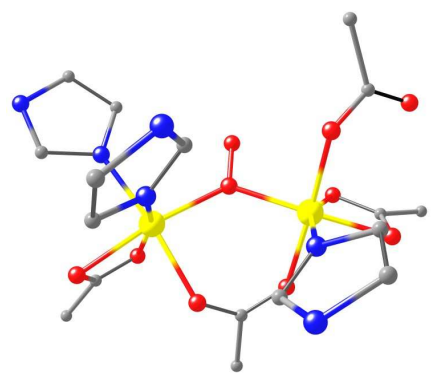

${ }_{227}$ M3- $\mu$-1,2- peroxo- $\mathrm{Fe}_{2}{ }^{\text {III/III }}$ with glutamate 227 protonated

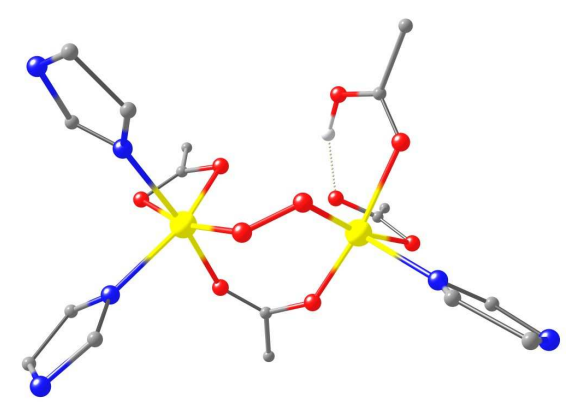

\section{Hydroperxo models:}

M1P. $\mu-\eta^{2}: \eta^{2}$ - hydroperoxo-Fe ${ }^{\text {IIIIII }}$

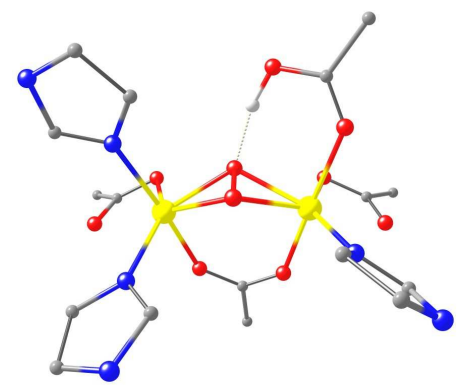

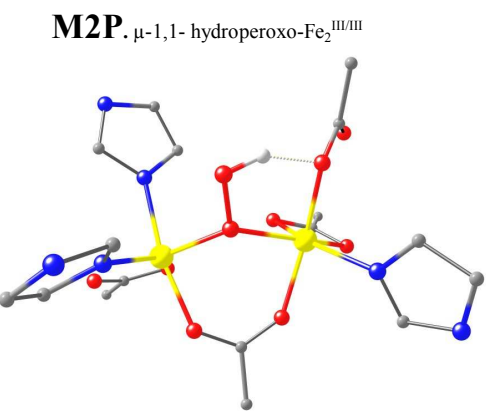




\section{Hydroperoxo models with protonated glutamate:}

227 M1P. $\mu-\eta^{2}: \eta^{2}$-hydroperoxo- $\mathrm{Fe}_{2}{ }^{\text {IIIIIII }}$ with glutamate 227 protonated

${ }_{227}$ M2P. $\mu-1,1$ - hydroperoxo- $\mathrm{Fe}_{2}{ }^{\text {IIIIIII }}$ with glutamate 227 protonated
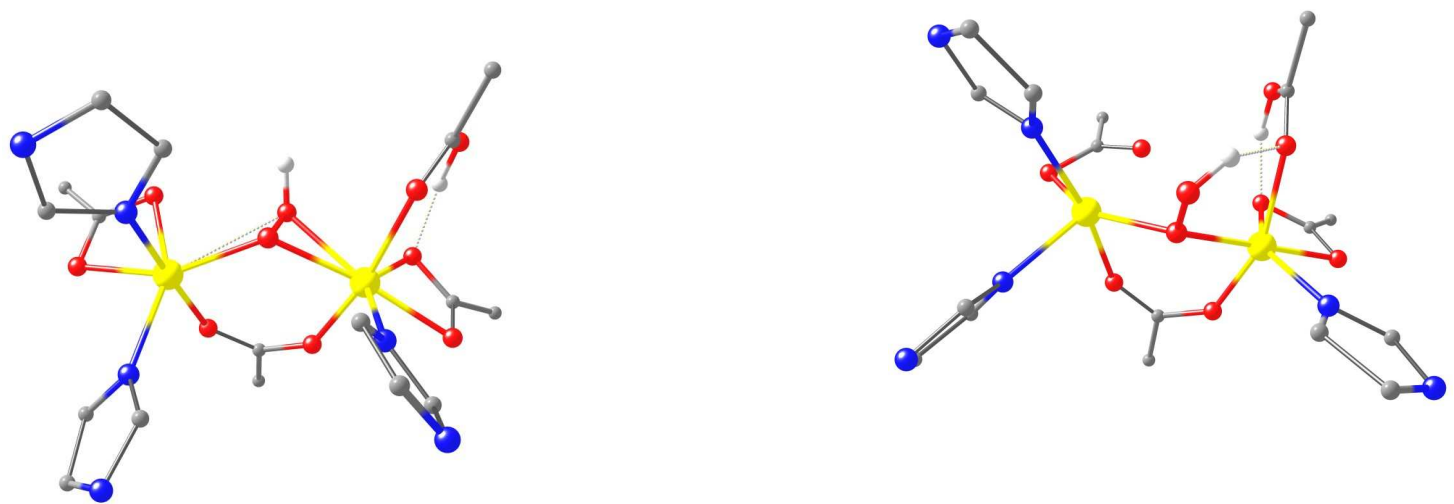

${ }_{196} \mathbf{M 3}^{`}$ - $\mu-1,2$ - hydroperoxo- $\mathrm{Fe}_{2}{ }^{\text {III/III }}$ with glutamate 196 protonated

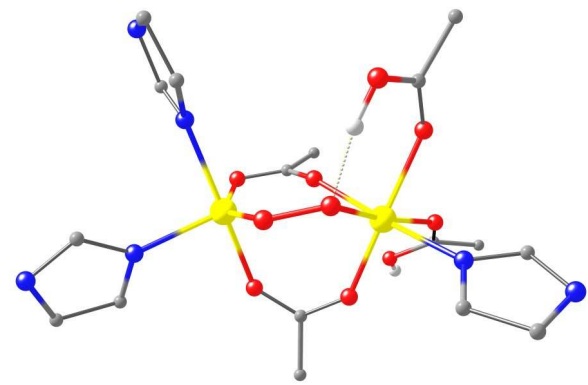


Table S1. B3LYP-D2 optimized bond lengths ( $\AA$ ) of the peroxo- $\mathrm{Fe}_{2}{ }^{\mathrm{II} / I I I}$ models.

\begin{tabular}{|c|c|c|c|c|c|c|c|c|c|c|c|}
\hline \multirow[t]{2}{*}{ Bond length } & \multirow[t]{2}{*}{ M1 } & \multirow[t]{2}{*}{ M2 } & \multirow[t]{2}{*}{ M3 } & \multirow[t]{2}{*}{${ }_{227} \mathrm{M} 1$} & \multirow[t]{2}{*}{${ }_{227} \mathrm{M} 2$} & \multirow[t]{2}{*}{${ }_{227} \mathrm{M} 3$} & \multirow[t]{2}{*}{ X-ray ${ }^{1}$} & \multicolumn{3}{|c|}{ Calculated value of $\mathrm{MMO}^{2,3}$ model } & \multirow[b]{2}{*}{$\mu-1,1^{4}$} \\
\hline & & & & & & & & $\begin{array}{l}\mu-\eta^{2}: \eta^{2} \text { (for R2- } \\
\text { wt) }\end{array}$ & $\begin{array}{l}\text { trans } \\
\mu-1,2^{3}\end{array}$ & $\begin{array}{l}\text { cis } \\
\mu-1,2^{3}\end{array}$ & \\
\hline $\mathrm{Fe} 1^{\mathrm{a}}-\mathrm{Fe} 2^{\mathrm{b}}$ & 3.483 & 3.702 & 4.023 & 3.483 & 3.410 & 3.568 & 3.503 & 3.607 & & 4.02 & \\
\hline $\mathrm{Fe} 1-\mu \mathrm{O} 1^{\mathrm{c}}$ & 2.074 & 2.142 & 1.855 & 2.112 & 1.970 & 1.797 & 1.942 & $2.092(2.01)$ & 1.87 & 1.92 & \\
\hline $\mathrm{Fe} 2-\mu \mathrm{O} 1^{\mathrm{c}}$ & 2.068 & 2.061 & 2.872 & 2.045 & 2.925 & 2.852 & 1.986 & $2.128(2.12)$ & - & - & \\
\hline $\mathrm{Fe} 1-\mu \mathrm{O} 2^{\mathrm{c}}$ & 1.992 & 3.054 & 2.840 & 2.016 & 2.080 & 2.724 & - & $1.939(1.96)$ & - & - & \\
\hline $\mathrm{Fe} 2-\mu \mathrm{O} 2^{\mathrm{c}}$ & 2.091 & 2.835 & 1.920 & 2.026 & 1.927 & 1.818 & - & $1.992(2.11)$ & 1.89 & 1.92 & \\
\hline$\mu \mathrm{O} 1-\mu \mathrm{O} 2^{\mathrm{c}}$ & 1.452 & 1.312 & 1.361 & 1.453 & 1.419 & 1.298 & - & $1.487(1.44)$ & 1.36 & 1.33 & 1.45 \\
\hline Fe1-O GLU101 & 2.204 & 2.212 & 2.336 & 2.119 & 2.118 & 2.051 & 2.056 & & & & \\
\hline Fe1-N His139 & 2.178 & 2.163 & 2.170 & 2.149 & 2.144 & 2.011 & 2.312 & & & & \\
\hline Fe1-N His223 & 2.162 & 2.154 & 2.127 & 2.154 & 2.140 & 1.983 & 2.275 & & & & \\
\hline Fe2-O Glu196 & 2.175 & 1.939 & 1.947 & 2.185 & 2.196 & 2.003 & 1.908 & & & & \\
\hline Fe2-N His 230 & 2.195 & 2.157 & 2.205 & 2.127 & 2.078 & 1.982 & 2.148 & & & & \\
\hline Fe2-O1 GLU227 & 1.932 & 2.186 & 2.219 & 2.140 & 2.111 & 1.992 & 1.960 & & & & \\
\hline Fe2-O2 GLU227 & 3.416 & 2.067 & 2.093 & 3.384 & 3.482 & 3.330 & 2.716 & & & & \\
\hline Fe1-OGlu136 (brig) & 2.023 & 2.080 & 2.077 & 2.017 & 2.051 & 1.941 & 2.018 & & & & \\
\hline Fe2-O Glu136(brig) & 2.105 & 2.035 & 2.054 & 1.981 & 1.962 & 1.925 & 1.992 & & & & \\
\hline Fe1-O1-O2 & 66.08 & 122.50 & 123.33 & 65.91 & 73.69 & 122.44 & & & & & \\
\hline$\Phi(\mathrm{Fe} 1-\mathrm{O} 1-\mathrm{O} 2-\mathrm{Fe} 2)$ & -129.66 & 166.25 & 86.47 & -130.75 & 107.38 & -32.55 & & (145) & 155 & 51 & \\
\hline
\end{tabular}

${ }^{a} \mathrm{Fe} 1$ - Metal Site containing two terminal histidine (HIS139, HIS223) and one glutamate (GLU101) residues.

${ }^{\mathrm{b}} \mathrm{Fe} 2$ - Metal Site containing one terminal histidine (HIS230) and two glutamate (GLU196, GLU227) residues.

${ }^{\mathrm{c}}$-Numbering as per figure $\mathrm{S} 1$. 
Table S2. B3LYP-D2 optimized bond lengths $(\AA)$ of the hydroperoxo-Fe ${ }_{2}{ }^{\mathrm{III} / \mathrm{III}}$ models

\begin{tabular}{|c|c|c|c|c|c|c|c|c|c|c|c|}
\hline \multirow[t]{2}{*}{ Bond length } & \multirow[t]{2}{*}{ M1P } & \multirow[t]{2}{*}{ M2P } & \multirow[t]{2}{*}{ M3P } & \multirow[t]{2}{*}{${ }_{227} \mathrm{M} 1 \mathrm{P}$} & \multirow[t]{2}{*}{${ }_{227} \mathrm{M} 2 \mathrm{P}$} & \multirow[t]{2}{*}{ 227M3P } & \multirow[t]{2}{*}{ X-ray ${ }^{1}$} & \multicolumn{3}{|c|}{ Calculated value of $\mathrm{MMO}^{2,3}$ model } & \multirow[b]{2}{*}{$\mu-1,1^{4}$} \\
\hline & & & & & & & & $\begin{array}{l}\mu-\eta^{2}: \eta^{2} \\
\text { (for R2-wt) }{ }^{2}\end{array}$ & $\begin{array}{l}\text { trans } \mu- \\
1,2^{3}\end{array}$ & cis $\mu-1,2^{3}$ & \\
\hline $\mathrm{Fe}^{\mathrm{a}}-\mathrm{Fe} 2^{\mathrm{b}}$ & 3.532 & 3.463 & 3.670 & 3.707 & 3.488 & 3.894 & 3.503 & 3.607 & & 4.02 & \\
\hline $\mathrm{Fe} 1-\mu \mathrm{O} 1^{\mathrm{c}}$ & 2.112 & 2.031 & 1.876 & 2.051 & 2.001 & 2.169 & 1.942 & $2.092(2.01)$ & 1.87 & 1.92 & \\
\hline $\mathrm{Fe} 2-\mu \mathrm{O} 1^{\mathrm{c}}$ & 2.069 & 2.031 & 3.420 & 2.089 & 2.086 & 2.916 & 1.986 & $2.128(2.12)$ & - & - & \\
\hline $\mathrm{Fe} 1-\mu \mathrm{O} 2^{\mathrm{c}}$ & 2.044 & 2.941 & 2.779 & 2.846 & 2.902 & 3.000 & - & $1.939(1.96)$ & - & - & \\
\hline $\mathrm{Fe} 2-\mu \mathrm{O} 2^{\mathrm{c}}$ & 2.087 & 2.941 & 2.662 & 2.238 & 2.990 & 1.958 & - & $1.992(2.11)$ & 1.89 & 1.92 & \\
\hline$\mu \mathrm{O} 1-\mu \mathrm{O} 2^{\mathrm{c}}$ & 1.460 & 1.433 & 1.414 & 1.449 & 1.426 & 1.437 & - & $1.487(1.44)$ & 1.36 & 1.33 & 1.45 \\
\hline$\mu \mathrm{O} 2-\mathrm{H}^{\mathrm{d}}$ & 1.562 & 0.993 & 0.974 & 0.975 & 0.983 & 0.973 & - & & & & \\
\hline Fe1-O GLU101 & 2.111 & 2.116 & 2.112 & 2.065 & 2.099 & 2.122 & 2.056 & & & & \\
\hline Fe1-N His139 & 2.149 & 2.092 & 2.165 & 2.115 & 2.139 & 2.120 & 2.312 & & & & \\
\hline Fe1-N His223 & 2.150 & 2.118 & 2.093 & 2.125 & 2.098 & 2.098 & 2.275 & & & & \\
\hline Fe2-O Glu196 & 2.147 & 2.098 & 2.119 & 2.137 & 2.133 & 2.168 & 1.908 & & & & \\
\hline Fe2-N His 230 & 2.139 & 2.112 & 2.171 & 2.104 & 2.083 & 2.070 & 2.148 & & & & \\
\hline Fe2-O1 GLU227 & 2.126 & 1.995 & 1.869 & 2.080 & 2.085 & 2.051 & 1.960 & & & & \\
\hline Fe2-O2 GLU227 & 3.433 & 3.537 & 3.690 & 3.472 & 3.462 & 3.442 & 2.716 & & & & \\
\hline Fe1-O Glu136 (brig) & 2.012 & 1.992 & 2.032 & 1.992 & 2.025 & 1.942 & 2.018 & & & & \\
\hline Fe2-O Glu136(brig) & 1.982 & 2.034 & 2.025 & 1.952 & 1.930 & 1.998 & 1.992 & & & & \\
\hline Fe1-O1-O2 & 66.90 & 115.15 & 114.562 & 107.52 & 114.63 & 111.02 & & & & & \\
\hline$\Phi(\mathrm{Fe} 1-\mathrm{O} 1-\mathrm{O} 2-\mathrm{Fe} 2)$ & -130.43 & -140.73 & -50.79 & -124.98 & -140.76 & -79.14 & & (145) & 155 & 51 & \\
\hline
\end{tabular}

${ }^{a} \mathrm{Fe} 1-$ Metal Site containing two terminal histidine (HIS139, HIS223) and one glutamate (GLU101) residues.

${ }^{\mathrm{b}} \mathrm{Fe} 2$ - Metal Site containing one terminal histidine (HIS230) and two glutamate (GLU196, GLU227) residues.

${ }^{c}-$ Numbering as per figure $\mathrm{S} 1$.

${ }^{\mathrm{d}} \mathrm{H}$ - hydrogen of the peroxo group 
Table S3. B3LYP optimized bond lengths $(\AA)$ of the peroxo-Fe ${ }_{2}^{\mathrm{III} / \mathrm{III}}$ models.

\begin{tabular}{|c|c|c|c|c|c|c|c|c|c|}
\hline \multirow[t]{2}{*}{ Bond length } & \multirow[t]{2}{*}{ M1 } & \multirow[t]{2}{*}{${ }_{227} \mathrm{M} 1$} & \multirow[t]{2}{*}{ M2 } & \multirow[t]{2}{*}{${ }_{227} \mathrm{M} 3$} & \multirow[t]{2}{*}{ X-ray ${ }^{1}$} & \multicolumn{3}{|c|}{ Calculated value of $\mathrm{MMO}^{2,3}$ model } & \multirow[b]{2}{*}{$\mu-1,1^{4}$} \\
\hline & & & & & & $\begin{array}{l}\mu-\eta^{2}: \eta^{22} \text { (for R2- } \\
w t)^{2}\end{array}$ & $\begin{array}{l}\text { trans } \mu- \\
1,2^{3}\end{array}$ & $\begin{array}{l}\text { cis } \\
\mu-1,2^{3}\end{array}$ & \\
\hline $\mathrm{Fe} 1-\mu \mathrm{O} 1^{\mathrm{c}}$ & 2.058 & 2.140 & 2.200 & 1.875 & 1.942 & $2.092(2.01)$ & 1.87 & 1.92 & \\
\hline $\mathrm{Fe} 2-\mu \mathrm{O} 1^{\mathrm{c}}$ & 2.122 & 2.068 & 2.108 & 2.880 & 1.986 & $2.128(2.12)$ & - & - & \\
\hline$\mu \mathrm{O} 1-\mu \mathrm{O} 2^{\mathrm{c}}$ & 1.455 & 1.450 & 1.307 & 1.356 & - & $1.487(1.44)$ & 1.36 & 1.33 & 1.45 \\
\hline Fe1-O GLU101 & 2.162 & 2.161 & 2.369 & 2.213 & 2.056 & & & & \\
\hline Fe1-N His139 & 2.206 & 2.172 & 2.210 & 2.180 & 2.312 & & & & \\
\hline Fe1-N His223 & 2.210 & 2.180 & 2.165 & 2.142 & 2.275 & & & & \\
\hline Fe2-O2 GLU227 & 2.101 & 2.174 & 2.065 & 2.164 & 2.716 & & & & \\
\hline Fe1-O Glu136 (brig) & 2.002 & 2.022 & 2.150 & 2.073 & 2.018 & & & & \\
\hline Fe2-O Glu136(brig) & 2.115 & 1.989 & 2.018 & 1.968 & 1.992 & & & & \\
\hline Fe1-O1-O2 & 71.273 & 65.036 & 116.198 & 120.426 & & & & & \\
\hline$\Phi(\mathrm{Fe} 1-\mathrm{O} 1-\mathrm{O} 2-\mathrm{Fe} 2)$ & 136.047 & 131.448 & 167.12 & 72.438 & & (145) & 155 & 51 & \\
\hline
\end{tabular}

${ }^{a} \mathrm{Fe} 1-$ Metal Site containing two terminal histidine (HIS139, HIS223) and one glutamate (GLU101) residues.

${ }^{\mathrm{b}} \mathrm{Fe} 2$ - Metal Site containing one terminal histidine (HIS230) and two glutamate (GLU196, GLU227) residues.

${ }^{\mathrm{c}}$-Numbering as per figure S1. 
Table S4. B3LYP optimized bond lengths $(\AA)$ of the hydroperoxo-Fe ${ }_{2}{ }^{\mathrm{III} / \mathrm{III}}$ models

\begin{tabular}{|c|c|c|c|c|c|c|c|c|c|c|}
\hline \multirow[t]{2}{*}{ Bond length } & \multirow[t]{2}{*}{ M1P } & \multirow[t]{2}{*}{${ }_{227} \mathrm{M} 1 \mathrm{P}$} & \multirow[t]{2}{*}{ M2P } & \multirow[t]{2}{*}{${ }_{227} \mathrm{M} 2 \mathrm{P}$} & \multirow[t]{2}{*}{${ }_{227} \mathrm{M}^{\prime}$} & \multirow[t]{2}{*}{ X-ray ${ }^{1}$} & \multicolumn{3}{|c|}{ Calculated value of $\mathrm{MMO}^{2,3}$ model } & \multirow[b]{2}{*}{$\mu-1,1^{4}$} \\
\hline & & & & & & & $\mu-\eta^{2}: \eta^{2}$ (for R2-wt) $)^{2}$ & trans $\mu-1,2^{3}$ & $\begin{array}{l}\text { cis } \\
\mu-1,2^{3}\end{array}$ & \\
\hline $\mathrm{Fe} 1^{\mathrm{a}}-\mathrm{Fe} 2^{\mathrm{b}}$ & 3.569 & 3.844 & 3.595 & 3.602 & 3.512 & 3.503 & 3.607 & & 4.02 & \\
\hline $\mathrm{Fe} 1-\mu \mathrm{O} 1^{\mathrm{c}}$ & 2.132 & 2.074 & 2.005 & 2.024 & 1.875 & 1.942 & $2.092(2.01)$ & 1.87 & 1.92 & \\
\hline $\mathrm{Fe} 2-\mu \mathrm{O} 1^{\mathrm{c}}$ & 2.096 & 2.138 & 2.071 & 2.069 & 2.876 & 1.986 & $2.128(2.12)$ & - & - & \\
\hline $\mathrm{Fe} 1-\mu \mathrm{O} 2^{\mathrm{c}}$ & 2.044 & 2.835 & 2.897 & 2.891 & 2.872 & - & $1.939(1.96)$ & - & - & \\
\hline $\mathrm{Fe} 2-\mu \mathrm{O} 2^{\mathrm{c}}$ & 2.090 & 2.211 & 2.983 & 2.966 & 1.939 & - & $1.992(2.11)$ & 1.89 & 1.92 & \\
\hline$\mu \mathrm{O} 1-\mu \mathrm{O} 2^{\mathrm{c}}$ & 1.458 & 1.450 & 1.432 & 1.430 & 1.360 & - & $1.487(1.44)$ & 1.36 & 1.33 & 1.45 \\
\hline$\mu \mathrm{O} 2-\mathrm{H}^{\mathrm{d}}$ & 1.594 & 0.975 & 0.996 & 0.983 & 1.644 & - & & & & \\
\hline Fe1-O GLU101 & 2.166 & 2.093 & 2.141 & 2.080 & 2.117 & 2.056 & & & & \\
\hline Fe1-N His139 & 2.178 & 2.136 & 2.134 & 2.151 & 2.152 & 2.312 & & & & \\
\hline Fe1-N His223 & 2.179 & 2.144 & 2.120 & 2.125 & 2.100 & 2.275 & & & & \\
\hline Fe2-O Glu196 & 2.139 & 2.096 & 1.975 & 2.120 & 2.109 & 1.908 & & & & \\
\hline Fe2-N His 230 & 2.154 & 2.115 & 2.134 & 2.081 & 2.116 & 2.148 & & & & \\
\hline Fe2-O2 GLU227 & 2.107 & 2.134 & 2.086 & 2.151 & 3.588 & 2.716 & & & & \\
\hline $\begin{array}{ll}\text { Fe1-O } & \text { Glu136 } \\
\text { (brig) } & \end{array}$ & 2.016 & 1.993 & 1.996 & 2.042 & 2.050 & 2.018 & & & & \\
\hline Fe2-O Glu136(brig) & 1.988 & 1.957 & 2.051 & 1.934 & 1.982 & 1.992 & & & & \\
\hline$\Phi(\mathrm{Fe} 1-\mathrm{O} 1-\mathrm{O} 2-\mathrm{Fe} 2)$ & 131.244 & 129.545 & 152.321 & 147.638 & 31.580 & & (145) & 155 & 51 & \\
\hline
\end{tabular}

${ }^{a} \mathrm{Fe} 1-$ Metal Site containing two terminal histidine (HIS139, HIS223) and one glutamate (GLU101) residues.

${ }^{\mathrm{b}} \mathrm{Fe} 2$ - Metal Site containing one terminal histidine (HIS230) and two glutamate (GLU196, GLU227) residues.

${ }^{\mathrm{c}}$-Numbering as per figure $\mathrm{S} 1$.

${ }^{\mathrm{d}} \mathrm{H}$ - hydrogen of the peroxo group 
Table S5. Calculate and experimental ${ }^{5}$ isomer shift $\delta(\mathrm{mm} / \mathrm{s})$, quadrupole splitting $\Delta \mathrm{Eq}(\mathrm{mm} / \mathrm{s})$, Net spin Population (NSP) of peroxo and hydroperoxo model complexes investigated. (B3LYP optimised structure used for spectroscopic property study)

\begin{tabular}{|c|c|c|c|c|c|c|c|c|c|c|c|c|c|}
\hline \multicolumn{7}{|c|}{ peroxo- $\mathrm{Fe}_{2}{ }^{\mathrm{III} / I I}$ model complexes } & \multirow[t]{2}{*}{$\operatorname{Exp}^{5}$} & \multicolumn{6}{|c|}{ Hydroperoxo- $\mathrm{Fe}_{2}{ }^{\mathrm{IIIIII}}$ model complexes } \\
\hline & M1 & M2 & M3 & ${ }_{227} \mathrm{M} 1$ & ${ }_{227} \mathrm{M} 2$ & ${ }_{227} \mathrm{M} 3$ & & M1P & M2P & M3P & ${ }_{227} \mathrm{M} 1 \mathrm{P}$ & ${ }_{227} \mathrm{M} 2 \mathrm{P}$ & ${ }_{227} \mathrm{M}^{\prime}$ \\
\hline \multicolumn{14}{|c|}{ Net spin Population } \\
\hline NSP (Fe1) & 3.96 & 3.97 & - & 3.95 & - & 3.88 & - & 3.98 & 4.04 & - & 4.01 & 4.03 & 3.96 \\
\hline NSP (Fe2) & -3.95 & -3.82 & - & -3.96 & - & -3.89 & - & -3.99 & -4.0 & - & -4.05 & -4.05 & -3.88 \\
\hline $\mathrm{NSP}(\mu-\mathrm{O} 1)$ & -0.05 & -0.14 & - & 0.04 & - & -0.22 & - & 0.15 & -0.0 & - & -0.00 & -0.11 & -0.27 \\
\hline $\operatorname{NSP}(\mu-\mathrm{O} 2)$ & -0.04 & -0.25 & - & 0.00 & - & 0.20 & - & -0.00 & -0.00 & - & 0.07 & -0.00 & 0.14 \\
\hline \multicolumn{14}{|c|}{ Spectroscopic parameters } \\
\hline$\delta(\mathrm{Fe} 1)$ & 0.75 & 0.68 & - & 0.72 & - & 0.71 & 0.54 & 0.75 & 0.62 & - & 0.73 & 0.64 & 0.75 \\
\hline$\delta(\mathrm{Fe} 2)$ & 0.75 & 0.68 & - & 0.78 & - & 0.64 & 0.61 & 0.72 & 0.58 & - & 0.58 & 0.59 & 0.64 \\
\hline$\Delta \mathrm{Eq}(\mathrm{Fe} 1)$ & 0.47 & 0.90 & - & -0.72 & - & 1.50 & -0.66 & -.67 & -0.28 & - & -0.72 & 0.70 & 0.67 \\
\hline$\Delta \mathrm{Eq}(\mathrm{Fe} 2)$ & 0.51 & -0.70 & - & 0.92 & - & 1.07 & 0.35 & 1.02 & -0.59 & - & 0.57 & 0.77 & 1.05 \\
\hline
\end{tabular}

Table 6. Calculated Zero-point corrected relative energy and heisenberg coupling constant $\mathrm{J}\left(\mathrm{cm}^{-}\right.$ ${ }^{1}$ ) of peroxo diiron(III) and hydroperoxo diiron(III) model complexes investigated. B3LYP-D2 relative energies $(\mathrm{kJ} / \mathrm{mol})$ are also shown .

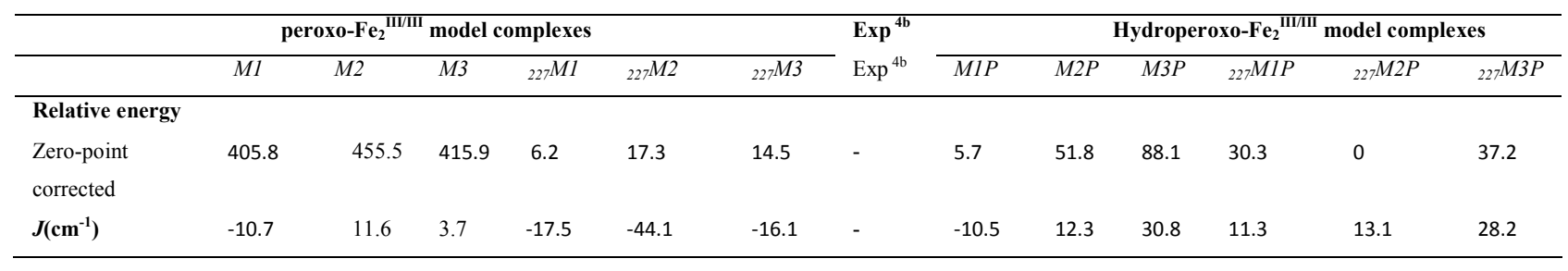


Calculation of exchange coupling for the M2 model: For the M2 model, the oxygen atoms are found to gain significant spin densities, particularly on the distal oxygen atom (see Figure $2 b$ ). Due to the radical nature of the bridging group, there are three different exchange interactions $J_{1}$ $J_{3}$ as defined in Figure $2 \mathrm{~b}$. The Fe-Fe exchange is described by $J_{1}$ interaction and asymmetric nature of the bridging oxygens lead to different $\mathrm{Fe}(1)$-radical and $\mathrm{Fe}(2)$-radical exchange parameters. Here the $J_{1}$ interaction is estimated to be weakly antiferromagnetic while the $J_{2}$ and $J_{3}$ interactions are found to be strongly antiferromagnetic in nature $\left(-119.5 \mathrm{~cm}^{-1}\right.$ and $\left.-365.5 \mathrm{~cm}^{-1}\right)$. The computed Fe-radical exchange interactions are consistent with the $J$ values reported for other Fe-radical centers. 
References:

(1) Choi, Y. S.; Zhang, H.; Brunzelle, J. S.; Nair, S. K.; Zhao, H. Proc. Natl. Acad. Sci. U. S. A., Early Ed.2008, 105, 6858-6863.

(2) Gherman, B. F.; Baik, M. H.; Lippard, S. J.; Friesner, R. A. J. Am. Chem. Soc. 2004, 126, 2978-2990.

(3) Rinaldo, D.; Philipp, D. M.; Lippard, S. J.; Friesner, R.A. J. Am. Chem. Soc. 2007, 129, 3135-3147.

(4) Jayapal, P.; Sundararajan, M.; Hillier, I. H.; Burton, N. A. Phys. Chem. Chem. Phys. 2006, 8, 4086-4094.

(5) Korboukh, V. K.; Li, N.; Barr, E.W.; Bollinger Jr, J. M.; Krebs, C. J. Am. Chem. Soc. 2009, $131,13608-13609$. 